\title{
On BLR Size Estimates in Reverberation Models
}

\author{
Alexander V. Melnikov and Ivan I. Shevchenko \\ Pulkovo Observatory of the Russian Academy of Sciences, \\ Pulkovskoje Ave. 65, St. Petersburg 196140, Russia \\ Email: melnikov, iis@gao.spb.ru
}

Keywords. galaxies: nuclei, galaxies: Seyfert, quasars: emission lines

Following the approach of Melnikov \& Shevchenko (2008), we explore how the nonlinearity in the emission-line luminosity $L_{1}$ of a broad-line region cloud, in its dependence on the ionizing continuum flux $F_{\mathrm{i}}$ incident on the cloud, affects estimates of the size of the broad-line region by means of cross-correlation methods. We show that the estimates obtained by straightforward cross-correlation of emission-line and continuum light curves can significantly underestimate the BLR size. We demonstrate examples of direct reverberation modelling of AGN emission-line light curves taking into account the nonlinearity of the " $L_{1}-F_{\mathrm{i}}$ " relation. This nonlinearity allows one to explain the differences in the time lags for different lines. Cross-correlation estimates of the BLR size turn out to be small in comparison to the estimates obtained by the direct reverberation modelling.

The mass of the black hole (BH) in the center of the BLR is usually calculated by the formula (see, e.g., Bentz et al. 2006) $M_{\mathrm{BH}} \approx f c \tau \Delta V^{2} / G \approx f R \Delta V^{2} / G$, where $\tau$ is the time lag for an emission line, $R$ is the BLR radius, $\Delta V$ is the width of the emission line, $c$ is the speed of light, and $G$ is the gravitational constant. The factor $f$ depends on the geometry, inclination, and dynamics of the BLR. Here the BLR radius is set to be equal to $c \tau$, as it is commonly done; the uncertainty is included in $f$. Note that this formula is valid, if the virial theorem is valid: in particular, the size of the BLR should be constant.

A hypothesis on possible variability of the BLR size of NGC 4151 was put forward by Kaspi et al. (1996), Peterson et al. (2002), and other researchers on the basis of cross-correlation analysis of optical spectral variability data at different time intervals of observations. Our reverberation modelling of the $\mathrm{H} \alpha$ light curve data of Kaspi et al. (1996) gives the value of the BLR radius matching the majority of the BLR size estimates of other authors. This removes necessity of any special physical interpretation of the small value of the cross-correlation time lag in $\mathrm{H} \alpha$ for these light curve data. In particular, the hypothesis by Kaspi et al. (1996) that the physical size of the BLR at the moment of their observations was an order of magnitude less than usual is not necessary. This strengthens the validity of the BH mass estimates, which are based on the validity of the virial theorem. Indeed, the dynamical timescale (determined from the width of the lines) in the BLR can be of the same order or even exceed the timescale of hypothetical variations of the BLR size (according to Kaspi et al. 1996, the latter timescale is less than 2000 days). If the size variability caused by external disturbances on such short timescales is absent, then the virial mass estimates are presumably valid.

\section{References}

Bentz, M. C., et al. 2006, ApJ, 651, 775

Kaspi, S., et al. 1996, ApJ, 470, 336

Melnikov, A. V., \& Shevchenko, I. I. 2008, MNRAS, 389, 478

Peterson, B. M., et al. 2002, ApJ, 581, 197 